\title{
NOTE TECHNIQUE \\ PRODUCTION DE BROCHETONS EN PETITS ETANGS PAR REPRODUCTION NATURELLE AMÉNAGÉE
}

\author{
C. BRY (1),Y. SOUCHON (2), G. NEVEU (3)
}

(1) I.N.R.A., Laboratoire de Physiologie des Poissons, 78350 - JOUY-EN-JOSAS.

(2) CEMAGREF-DQEPP, 3, quai Chauveau, 69009 - LYON.

(3) C.S.P., Centre du Paraclet, B.P. 5, 80440 - BOVES.

\begin{abstract}
RESUME
Nous présentons dans cette note le protocole d'obtention de familles de brochetons par reproduction naturelle pratiquée dans un milieu " aménagé " (petits étangs vidangeables, enherbés et peu profonds, dotés de poisson - fourrage, dépourvus de prédateurs et dont le niveau d'eau est stable). Des indications sont données sur les caractéristiques des étangs utilisés, la mise en charge (reproducteurs brochet et poisson-fourrage), les niveaux de production obtenus et le calendrier des opérations.
\end{abstract}

\section{TECHNICAL NOTE : PRODUCTION OF YOUNG NORTHERN PIKE IN SMALL PONDS FROM MANAGED NATURAL SPAWNING.}

\section{SUMMARY}

The present technical note describes a method to produce young northern pike (Esox Jucius) families by managed natural spawning in a " protected " environment (i.e. small, drainable, grassy and shallow ponds, containing forage-fish but no predator, and with a constant water level). Some indications on the features of the spawning ponds, the stocking rates (northern pike spawners and forage-fish), the production levels and the temporal organization are given.

\section{INTRODUCTION}

La reproduction naturelle aménagée du brochet, pratiquée en petits étangs, permet d'obtenir de façon simple des productions de brochetons selon un mode extensif. Cette méthode constitue une alternative intéressante à la production extensive de brochetons par alevinage classique, en particulier lorsqu'on ne dispose pas d'une écloserie. La technique est basée sur l'introduction d'une seule femelle par étang : La présence d'une seule ponte permet de régulariser la densité numérique des brochetons récoltés et de limiter. dans une certaine mesure, l'hétérogénéité de leur taille: ces caractéristiques résultent d'une relative homogénéité de la croissance initiale et de la possibilité de rationaliser la mise en charge. La reproduction naturelle aménagée constitue une version optimisée de la reproduction naturelle en milieu " sauvage " (voir la revue de SOUCHON. 1983). Les éléments de cette note technique sont fondés sur plusieurs années d'expérimentations dans la région de la Somme (Centre du Paraclet, Conseil Supérieur de la Pêche) et dans la Dombes (domaine de M. Verzier). Les résultats expérimentaux obtenus ont déjà fait l'objet de comptes rendus détaillés (BRY et SOUCHON. 1982 ; BRY et al. , 1983). En ce qui concerne la comparaison entre la reproduction naturelle aménagée et d'autres méthodes de reproduction, on se reportera au travail de TREBAOL (1982).

\section{I - CARACTÉRISTIQUES DES ETANGS}

\section{1 / Superficie}

La reproduction naturelle aménagée a été testée dans des étangs de surface variable (200 à $2000 \mathrm{~m}^{2}$ ). La superficie idéale pour un étang consacré au grossissement de fingerlings de brochet pourrait être de l'ordre de $1500 \mathrm{~m}^{2}$ : pour exploiter une surface donnée, des étangs plus petits peuvent être en effet plus onéreux du fait de la multiplication des ouvrages de vidange, tandis que des surfaces supérieures entraînent des durées de vidange particulièrement longues, surtout si la végétation est 
abondante, ce qui pénalise la récolte finale (accentuation de l'hétérogénéité de la taille finale des brochetons et du cannibalisme). D'autre part. de petits étangs de quelques centaines de $\mathrm{m}^{2}$ sont généralement plus productifs (par unité de surface) que des pièces d'eau de superficie relativement grande (un demi-hectare et plus; HUET et TIMMERMANS, 1958)

\section{2 / Morphologie}

Les étangs doivent être peu profonds (de 0,2 m dans la partie amont à $0,8-1,0 \mathrm{~m}$ en aval au voisinage de l'ouvrage de vidange). Des fossés de largeur approximative $0.5 \mathrm{~m}$ disposés le long des berges et éventuellement selon une diagonale peuvent faciliter les opérations de vidange. L'existence d'une zone suffisamment profonde $(0,8-1,0 \mathrm{~m})$ et large de quelques mètres dans la partie aval peut favoriser la survie des reproducteurs brochet après la fraie, en particulier dans les cas où une végétation très dense (macrophytes émergés par exemple) se développe en mai dans les parties les moins profondes.

\section{3 / Végétation}

La majeure partie de la superficie des étangs doit être bien pourvue en végétation. En ce qui concerne nos sites expérimentaux, la végétation se composait de graminées aquatiques (Pha/aris sp. Glyceria sp.), d'oseille aquatique (Rumex sp.) et d'herbes terrestres submergées au Paraclet (Fig. 1), et d'Elodée (Helodea canadensis) dans la Dombes. En fait, le brochet fait preuve d'un certain opportunisme dans le choix du substrat de ponte (voir McCARRAHER et THOMAS, 1972 et revue par SOUCHON, 1983), et des formations végétales très variées peuvent convenir à sa reproduction naturelle: graminées (Agrostis sp., Calamagrostis sp., Glyceria sp., etc.), jonc (Juncus bulbosus), cypéracées (Carex sp., Scirpus sp.), potamots (Potamogeton sp.), utriculaires (Utricularia sp.), myriophyllées et cératophyllées, plantes nageantes à tiges submergées comme le plantain d'eau (Alisma $\mathrm{sp}$.), ou encore tapis denses de végétation courte (Chara sp.), herbes terrestres de prairie ou de fossé inondés et débris végétaux divers. Pour la détermination de végétaux aquatiques, on pourra se référer à l'article de BARBE (1984).

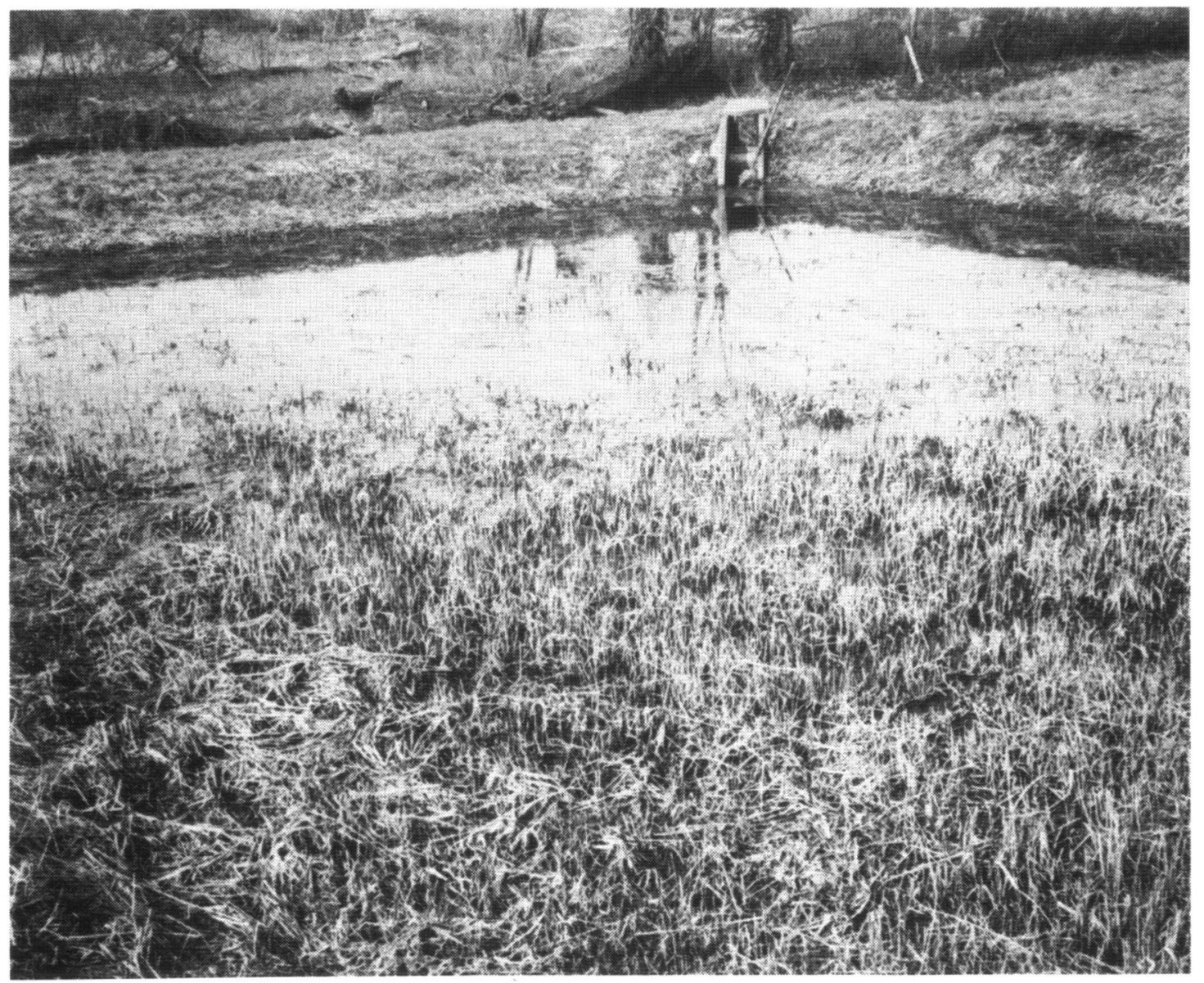

Figure 1: Petit étang à fond enherbé utilisé pour la production de brochetons par reproduction naturelle aménagée. 


\section{II - MISE EN CHARGE}

Les opérations de mise en charge et de vidange sont symbolisées au niveau de la figure 2. Le calendrier suggéré ici s'entend pour des étangs situés à basse altitude. Dans le cas des milieux d'altitude, le réchauffement des eaux au printemps est généralement plus tardif, et les opérations doivent s'envisager avec un retard de 15 jours à 2 mois selon les cas. Après un assec de 2 à 9 mois, les étangs sont mis en eau début février. Un dispositif de piégeage au niveau de l'arrivée d'eau évite l'introduction éventuelle d'espèces indésirables. Le niveau de l'eau doit rester stable, en particulier de fin février à début avril, pour éviter l'éventuelle mise à sec d'une partie de la ponte.

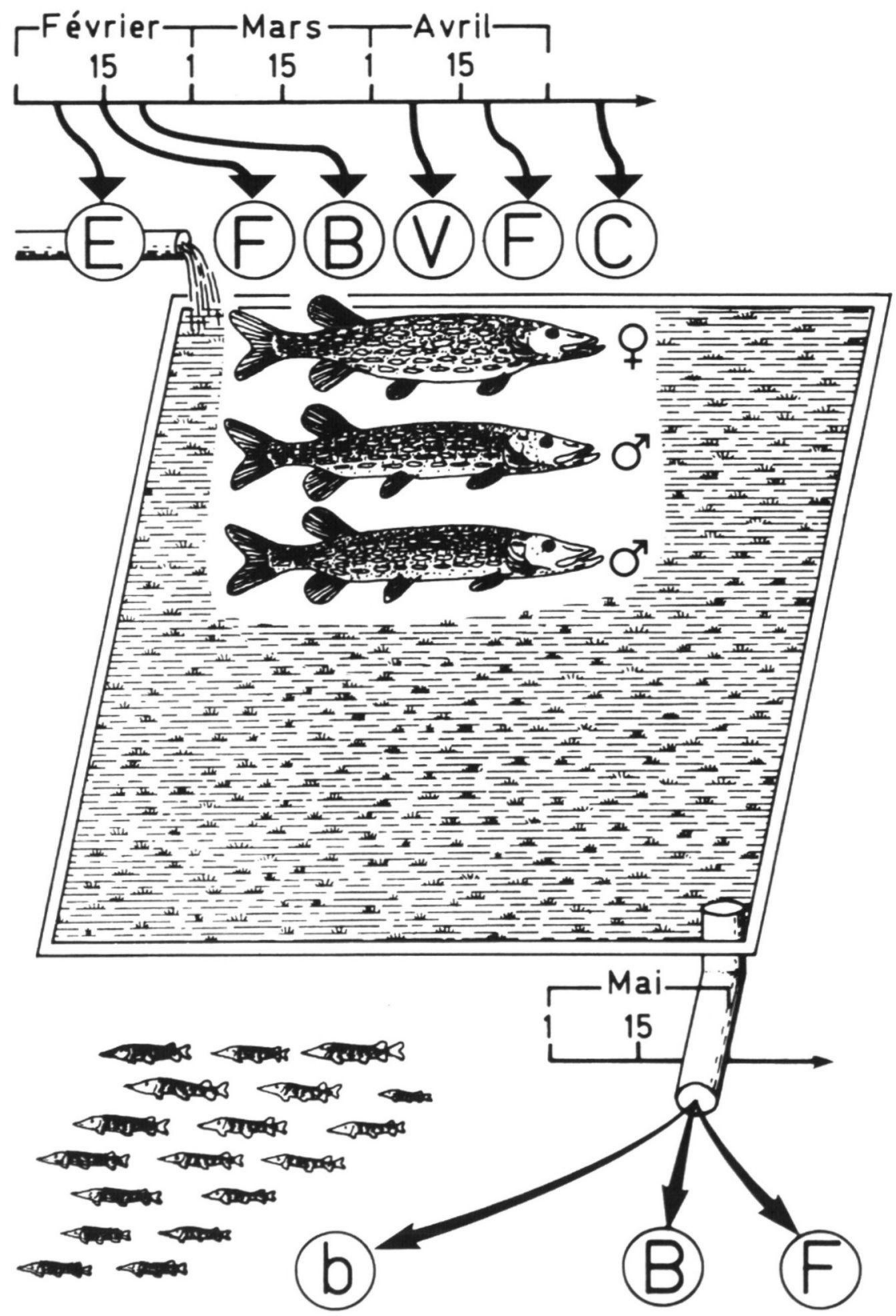

Figure 2: Représentation schématique de la production de brochetons par reproduction naturelle aménagée. Signification des symboles : $E=$ mise en eau : $F=$ poissonfourrage : $\mathbf{B}=$ brochets reproducteurs : $\mathbf{V}=$ vérification de la reproduction : $C=$ contrôle de la croissance des brochetons ; $b=$ brochetons.

Nota : les proportions relatives des dimensions de l'étang, de la taille des reproducteurs et de celle des brochetons n'ont pas été respectées dans ce diagramme. 


\section{1 / Poisson-fourrage}

Du poisson-fourrage de taille adéquate (15 à $30 \%$ de celle des géniteurs brochet) est introduit dans les étangs à raison de $0,2 \mathrm{~kg}$ par $\mathrm{kg}$ de brochet, soit quelques jours avant les géniteurs brochet (10 - 15 février), soit en même temps que ces derniers. Le choix du poisson-fourrage se portera sur des cyprinidés comme la brême commune (Abramis brama), le gardon (Rutilus rutilus), le rotengle (Scardinius erythrophthalmus) ou la tanche (Tinca tinca), en excluant les espèces susceptibles d'exercer une prédation importante sur de jeunes alevins, comme la perche commune (Perca fluviatilis).

Un deuxième apport de poisson-fourrage $(0,4 \mathrm{~kg}$ par $\mathrm{kg}$ de brochet) est réalisé à partir de la mi-avril, après la détection de brochetons mesurant une vingtaine de $\mathrm{mm}$. Le but de cette seconde introduction est de répondre aux exigences métaboliques des géniteurs brochet qui sont particulièrement importantes dans les semaines qui suivent la reproduction, en raison de la dépense d'énergie somatique liée à la fraie (DIANA, 1979 ; DIANA et MACKAY, 1979) et de l'élévation de la température de l'eau en avril - mai. Dans les régions où la température de l'eau des étangs au cours de la période mi-avril - fin mai est élevée (moyenne supérieure à $15^{\circ} \mathrm{C}$ ), cette seconde ration de poisson-fourrage pourra être doublée.

\section{2 / Reproducteurs brochet}

La mise en charge intervient à la mi-février, à raison d'une femelle et de deux mâles par étang. Les mâles sont aisément identifiés par l'émission de sperme suite à un léger massage pratiqué sous anesthésie au niveau de la région postérieure de l'abdomen. Pour la détermination des sexes, on pourra également faire appel à des critères morphologiques concernant la région urogénitale (CASSELMAN, 1974). La raison d'être du deuxième mâle est de permettre le déroulement de la reproduction dans l'hypothèse - peu probable —d'une mortalité précoce du premier. On peut noter à cet égard qu'au moment de la fraie en milieu " sauvage ". la femelle est le plus souvent accompagnée par deux ou trois mâles (CLARK, 1950). Pour limiter les possibilités de cannibalisme, les géniteurs destinés à un étang donné sont choisis de taille aussi homogène que possible : écart relatif inférieur à $10 \%$ entre les valeurs extrêmes des longueurs totales et inférieur à $20 \%$ pour les poids. La femelle doit avoir des ovaires correctement développés (abdomen d'aspect bombé).

Pour exploiter au mieux un étang de production de brochetons, il convient de pratiquer une mise en charge adéquate, que l'on travaille en alevinage classique (choix du nombre d'alevins introduits par $\mathrm{m}^{2}$ ) ou en reproduction naturelle aménagée (sélection du poids de la femelle destinée à un étang de superficie donnée). Une sous-charge excessive conduit à une sous-exploitation des ressources trophiques de l'étang, alors qu'une surcharge - même légère - peut provoquer une chute de la production numérique et une augmentation de l'hétérogénéité de la taille des brochetons récoltés. Le créneau de mise en charge optimum peut varier selon la région ou le type d'étang où l'on opère (variabilité des capacités d'accueil du milieu) et sa définition demande quelques tests préliminaires. A titre indicatif, le poids - en février - d'une femelle destinée à un étang de $1500 \mathrm{~m}^{2}$ peut être de l'ordre de $1750 \mathrm{~g}$ (longueur à la fourche: $60 \mathrm{~cm}$ environ).

En ce qui concerne les manipulations nécessaires à la détermination du sexe et du poids des géniteurs brochet, on prendra soin de les effectuer sous anesthésie (solution aqueuse de 2 phénoxyethanol à $0,5 \mathrm{ml} / \mathrm{l}$ ) et de façon aussi brève que possible.

\section{III. - CARACTÉRISTIQUES DE LA PRODUCTION}

Les alevins issus de la reproduction naturelle peuvent être détectés, selon les cas, entre la dernière semaine de mars et la mi-avril (alevins en cours de résorption ou nageants). En règle générale, les vidanges ont lieu au cours de la deuxième quinzaine de mai. Une vidange s'effectue de façon d'autant plus lente que la végétation est plus dense, pour permettre aux brochetons de descendre progressivement vers l'aval sans être piégés par les végétaux. A titre indicatif, la vidange d'un étang de $1500 \mathrm{~m}^{2}$ peut s'étaler sur 3 à 5 jours. Le choix de la période précise de vidange d'un étang donné dépend en fait de la taille moyenne à laquelle on souhaite récolter les brochetons. Une évaluation approximative de celle-ci à une date donnée peut être obtenue en mesurant une dizaine d'individus capturés à l'épuisette dans diverses parties de l'étang. En fin d'élevage (deuxième quinzaine de mai) et si la température de l'eau est élevée (valeur movenne supérieure à $15^{\circ} \mathrm{C}$ ), la vitesse de croissance des brochetons peut dépasser $2 \mathrm{~mm}$ par jour (FRANKLIN et SMITH, 1963) et un cannibalisme excessif peut alors se développer si la densité de proies de taille convenable devient insuffisante. Ce phénomène doit inciter à faire preuve d'une certaine vigilance et à ne pas effectuer de vidanges trop tardives

\section{$1 /$ Brochetons}

Dans un milieu favorable à la croissance du jeune brochet, c'est-à-dire caractérisé, entre autres, par la présence de zoo-plancton et d'invertébrés benthiques en densité et de taille appropriées. les productions sont en moyenne de l'ordre de 2 brochetons de $2.5 \mathrm{~g} \mathrm{par} \mathrm{m}^{2}$ (soit $50 \mathrm{~kg} / \mathrm{ha}$ ). De telles productions sont purement extensives, c'est-à-dire obtenues sans apport exogène. II est possible 
d'augmenter le niveau de production en introduisant de façon répétée du plancton collecté dans un autre plan d'eau ou en stimulant la production endogène de plancton par l'adjonction de fumure (LANOISELEE, 1984).

La date précise à laquelle le poids moyen des brochetons dans un étang atteint une valeur donnée dépend de différents facteurs : date de la ponte de la femelle, régime thermique de l'étang, adéquation entre la mise en charge et les ressources alimentaires. Lorsque l'on souhaite récolter des brochetons dont la vulnérabilité vis-à-vis de prédateurs ne soit pas trop importante, on retient parfois un poids moyen de l'ordre de $2,5 \mathrm{~g}$ (longueur totale de l'ordre de $80 \mathrm{~mm}$ ). Une telle taille est normalement atteinte avant la fin du mois de mai.

Une certaine hétérogénéité de la taille finale caractérise tout grossissement extensif de brochetons en étang. Cette hétérogénéité résulte fondamentalement de celle du milieu (variabilité intraétang de la température, de la densité de proies, de la nature des abris, etc.) mais aussi de différences entre individus pour ce qui concerne l'aptitude à la capture des proies (IVANOVA et LOPATKO, 1983). L'hétérogénéité finale peut être accentuée par une surcharge initiale et tend a augmenter dans le cas de récoltes tardives

\section{2 / Reproducteurs brochet}

Les reproducteurs sont capturés en même temps que les brochetons. En règle générale, leur taux de survie fin mai est élevé (de l'ordre de $80 \%$ pour les femelles et 60 \% pour les mâles), dans la mesure où la quantité et la taille du poisson-fourrage sont appropriées et où les géniteurs disposent d'une zone minimum de profondeur relativement importante dans la partie aval de l'étang. Le taux de survie après la reproduction peut chuter de façon importante si l'état initial des géniteurs en février n'est pas optimal (présence de quelques lésions superficielles d'étendue limitée par exemple). Il est extrêmement rare d'enregistrer une mortalité avant la fraie.

\section{3 / Poisson-fourrage}

Le taux de survie du poisson-fourrage, mesuré à la vidange de fin mai, est très variable et en moyenne de l'ordre de $40 \%$. Il dépend, entre autre facteurs, de la " capturabilité " du poissonfourrage par les géniteurs brochet. L'observation répétée d'une faible survie du poisson-fourrage suggère d'en augmenter les quantités introduites. La biomasse du poisson-fourrage récolté est souvent du même ordre que la biomasse introduite, compte tenu de la croissance des individus survivants.

\section{REMERCIEMENTS}

Cette étude a été prise en charge par le Conseil Supérieur de la Pêche. Nos remerciements vont à Messieurs J.P.CAVITTE, J.M.JANTZEN et J.VERZIER pour l'accueil qu'ils nous ont réservé, à J. GALLE pour la qualité de l'illustration graphique, ainsi qu'à C. GILLET, H. LE LOUARN et G. MAISSE pour la lecture critique du manuscrit.

\section{BIBLIOGRAPHIE}

BARBE J., 1984. Les végétaux aquatiques: données biologiques et écologiques ; clés de détermination des macrophytes de France. Bull. Français Piscic., numéro spécial, Juin 1984.

BRY C. et SOUCHON Y., 1982. Production of young northern pike families in small ponds: natural spawning versus fry stocking. Trans. Am. Fish. Soc., 111,476-480.

BRY C., SOUCHON Y., NEVEU G., TREBAOL L., 1983. Production de familles de brochetons en petits étangs par reproduction naturelle aménagée : bilan de trois années d'expérimentation et comparaison avec la méthode d'alevinage. Bull. Français Piscic., 288, 46-56.

CASSELMAN J.-M., 1974. External sex determination of northern pike, Esox Jucius Linnaeus. Trans. Am. Fish. Soc., 103. 343-347. Également cité en détail p. 338-339, in R. Billard Ed., le Brochet : gestion dans le milieu naturel et élevage, INRA Publ., Paris.

CLARK C.F., 1950. Observations on the spawning habits of the northern pike Esox lucius, in northwestern Ohio. Copeja, 1950 (4). 285-288.

DIANA J.S., 1979. The feeding pattern and daily ration of a top carnivore, the northern pike (Esox lucius). Can. J. Zool., 57, 2121-2127.

DIANA J.S. et MACKAY W.C., 1979. Timing and magnitude of energy deposition and loss in the body, liver, and gonads of northern pike (Esox lucius). J. Fish. Res. Board Can., 36, 481-487

FRANKLIN D.R. et SMITH L.L., Jr, 1963. Early life history of the northern pike with special reference to the factors influencing the numerical strength of year classes. Trans. Am. Fish. Soc., 92, 91-110. 
HUET M. et TIMMERMANS J.A., 1958. Esociculture : production de brochetons de sept semaines. Trav. Stat. Rech. Eaux et Forêts, Sér. D. 24, 1 - 10.

IVANOVA M.N. et LOPATKO A.M., 1983. Feeding behavior of pike, Esox lucius, larvae from the progeny of a single pair of spawners. J./chtyol., 4, 171-173.

LANOISELEE B., 1984. Fertilisation organique en aquaculture : utilisation du lisier de porc pour l'alevinage de poissons d'étang : Thèse de docteur-ingénieur, I.N.A. Paris-Grignon, 252 p.

MCCARRAHER D.B. et THOMASR.E., 1972. Ecological significance of vegetation to northern pike, Esox lucius, spawning. Trans. Am. Fish. Soc., 101, 560-563.

SOUCHON Y., 1983. La reproduction du brochet(Esox /ucius) dans le milieu naturel : revue bibliographique, p. 21 -37, in R. Billard Ed., le Brochet : gestion dans le milieu naturel et élevage. INRA Publ., Paris.

TREBAOL L., 1982. L'élevage du brocheton de repeuplement : comparaison de trois systèmes de production. Rapport de fin d'études, I.N.A. Paris-Grignon, $100 \mathrm{p}$ 